\title{
Solving the Maximum Matching Problem on Bipartite Star123-Free Graphs in Linear Time
}

\author{
Ruzayn Quaddoura \\ Department of Computer Science, Faculty of Information Technology, Zarqa University, Zarqa, Jordan \\ Email: ruzayn@zu.edu.jo
}

Received 6 April 2015; accepted 23 January 2016; published 26 January 2016

Copyright (C) 2016 by author and Scientific Research Publishing Inc.

This work is licensed under the Creative Commons Attribution International License (CC BY).

http://creativecommons.org/licenses/by/4.0/

(c) (i) Open Access

\begin{abstract}
The bipartite Star $_{123}$-free graphs were introduced by V. Lozin in [1] to generalize some already known classes of bipartite graphs. In this paper, we extend to bipartite Star $_{123}$-free graphs a linear time algorithm of J. L. Fouquet, V. Giakoumakis and J. M. Vanherpe for finding a maximum matching in bipartite $\operatorname{Star}_{123}, P_{7}$-free graphs presented in [2]. Our algorithm is a solution of Lozin's conjecture.
\end{abstract}

\section{Keywords}

Bipartite Graphs, Decomposition of Graphs, Design and Analysis of Algorithms, Matching

\section{Introduction}

A matching $M$ of a graph $G=(V, E)$ is a subset of edges with the property that no two edges of $M$ share a common vertex. A matching is called induced if the subgraph of $G$ induced by $M$ consists of exactly $M$ itself. The maximum matching problem is to find a matching with the maximum cardinality. Graph matching is one of the fundamental problems in combinatorial optimization because of its use in various fields such as computational biology [3], pattern recognition [4], computer vision [5], music information retrieval [6], and computational music theory [7]. For arbitrary graphs, it is known that this problem can be solved in $O(m \sqrt{n})$ time [8]. Moitra and Johnson gave an $O(n \log n)$ time algorithm on interval graphs [9]. In addition Alt, Blum, Mehlhorn, and Paul gave an $O\left(n^{1.5} \sqrt{m \log n}\right)$ time algorithm on bipartite graphs [10]. In [11] Yu and Yang exhibited an $O(n)$ time algorithm for the maximum matching problem on cographs. This result was extended in [12] by Fouquet, Parfenoff and Thuillier to a wider class, namely the $P_{4}$-tidy graphs. Also the technique developed in [11] was used by Fouquet, Giakoumakis and Vanherpe in [2] to find an $O(n)$ time algorithm for the maximum matching problem on bipartite $P_{7}$, Star $_{123}$-free graphs (see Figure 1). In [1], Lozin studied the class of bipartite 


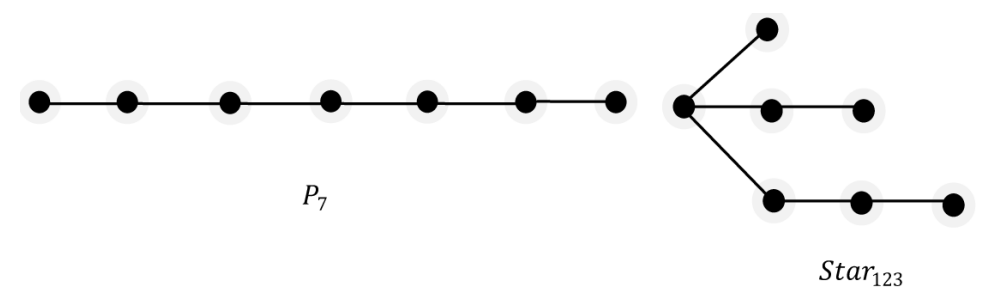

Figure 1. Forbidden configurations for $P_{7}$, Star $_{123}$-free graphs.

$\mathrm{Star}_{123}$-free graphs and conjectured that both maximum induced matching problem and maximum matching problem in this class can be solved in linear time. The first one has been solved in [13]. In this paper we shall solve Lozin's conjecture for maximum matching problem by extending the matching algorithm for the class of $P_{7}$, Star $_{123}$-free graphs [2] to the class of bipartite Star $_{123}$-free graphs. Our algorithm is based on the recognition algorithm of the class Star $_{123}$-free bipartite graphs introduced by Quaddoura in [14].

\section{Definitions and Properties}

For terms not defined in the paper the reader can refer to [15]. The graphs considered in this paper are finite without multiple edges and loops. As usual, for any graph $G$ we denote the set of its vertices by $V(G)$ and by $E(G)$ the set of its edges (or simply by $V$ and $E$ if there is no risk of confusion) and their respective cardinalities by $n$ and $m$. A bipartite graph $G=(B \cup W, E)$ is defined by two disjoint vertex subsets $B$ the black vertices and $W$ the white ones, and a set of edges $E \subseteq B \times W$. The bi-complement of a bipartite graph $G=(B \cup W, E)$ is the bipartite graph defined by $\bar{G}^{b i p}=(B \cup W, B \times W-E)$. If the color classes $B$ and $W$ are both non empty, the graph will be called bichromatic, monochromatic otherwise. A vertex $x$ will be called isolated (resp. universal) if $x$ has no neighbors in $G$ (resp. in $\bar{G}^{\text {bip }}$ ). A complete bipartite graph is a graph having only universal white vertices and universal black vertices. A stable set is a subset of pairwise non-adjacent vertices. A chordless path on $k$ vertices is denoted by $P_{k}$ and a chordless cycle on $k$ vertices is denoted by $C_{k}$. Given a subset $X$ of the vertex set $V(G)$, the subgraph induced by $X$ will be denoted by $G[X]$. A set $A \subseteq V$ is called a module if every vertex in $V-A$ is either adjacent to all vertices in $A$ or none of them. The representative graph of a graph $G$ is the subgraph of $G$ induced by the set of vertices containing one vertex from each proper maximal module of $G$. A graph $G$ is called $Z$-free where $Z$ is a set of graphs, when $G$ does not contain an induced subgraph isomorphic to a graph of $Z$.

Definition 1 [2]. Given a bipartite graph $G=(B \cup W, E)$ of order at least 2, $G$ is $K+S$ graph if and only if $G$ contains an isolated vertex or its vertex set can be decomposed into two sets $K$ and $S$ such that $K$ induces a complete bipartite graph while $S$ is a stable set.

Property 2 [2]. Let $G=(B \cup W, E)$ be a bipartite graph of order at least 2. $G$ is $K+S$ graph if and only if there exists a partition of its vertex set into two non empty classes $V_{1}$ and $V_{2}$ such that all possible edges exists between the black vertices of $V_{1}$ and the white vertices of $V_{2}$ while there is no edge connecting a white vertex of $V_{1}$ with a black vertex of $V_{2}$.

Such partition is referred as associated partition of $G$ and is denoted by the ordered pair $\left(V_{1}, V_{2}\right)$ [2].

Property 3 [2]. A bipartite graph $G$ is a $K+S$ graph if and only if $G$ admit a unique (up to isomorphism) partition of its vertex set $\left(V_{1} \cup V_{2} \cup \cdots \cup V_{k}\right)$ satisfying the following conditions:

1) $\forall i=1, \cdots, k-1,\left(V_{1} \cup \cdots \cup V_{i}, V_{i+1} \cup \cdots \cup V_{k}\right)$ is an associated partition to the graph $G$.

2) $\forall i=1, \cdots, k, G\left[V_{i}\right]$ is not a $K+S$ graph.

The partition $\left(V_{1}, \cdots, V_{k}\right)$ of the above property is called $K+S$ decomposition while a set $V_{i}$ said to be $K+S$ component of the graph.

From $K+S$ decomposition together with the decomposition of bipartite graph $G$ into its connected components (parallel decomposition) or those of $\bar{G}^{\text {bip }}$ (series decomposition) yield a new decomposition scheme for $G$ called canonical decomposition. It is shown in [2] that whatever the order in which the decomposition operators are applied ( $K+S$ decomposition, series decomposition or parallel decomposition), a unique set of indecomposable (or prime) graphs with respect to canonical decomposition is obtained. Obviously, a unique tree is 
associated to this decomposition. The internal nodes are labeled according to the type of decomposition applied, while every leaf correspond to a vertex of $G$. Hence there are four types of internal nodes, parallel node (labeled $P$ ), series node (labeled $S$ ), $K+S$ node (labeled $K+S$ ), and indecomposable node (labeled $N$ ). By convention, the set of vertices corresponding to the set of leafs having an internal node $\alpha$ as their least common ancestor will be denoted simply by $\alpha$.

Lozin in [1] gives the following characterization for bipartite Star $_{123}$-free graphs.

Theorem 4 [1]. Let $G$ be a bipartite Star ${ }_{123}$-free graph. One of the following hold.

1) $G$ is $K+S$ graph.

2) $G$ and $\bar{G}^{\text {bip }}$ aren't both connected.

3) The representative graph of $G$ or the bi-complement of the representative graph of $G$ is a path $P_{k}$ or a cycle $C_{k}$ with $k \geq 7$.

It is shown in [14] that the representative graph of a graph $G$ is a path $P_{k}$ or $\bar{P}_{k}^{\text {bip }}$ or a cycle $C_{k}$ or $\bar{C}_{k}^{\text {bip }}$ if and only if $G$ is an extended path $E P_{k}$ or a bi-complement of an extended path $E P_{k}$ or an extended cycle $E C_{k}$ or a bi-complement of an extended cycle $E C_{k}$ respectively. More precisely, (see Figure 2).

Definition 5 [14]. A graph $G$ is said to be an extended path $E P_{k}$ if there is a partition of the vertex set of $G$ into a monochromatic sets $\left\{V_{1}, \cdots, V_{k}\right\}$ such that $E=\bigcup_{i=1}^{k-1} V_{i} \times V_{i+1}$.

Definition 6 [14]. A graph $G$ is said to be an extended cycle $E C_{k}$ if there is a partition of the vertex set of $G$ into a monochromatic sets $\left\{V_{1}, \cdots, V_{k}\right\}$ such that $E=\bigcup_{i=1}^{k-1} V_{i} \times V_{i+1} \cup V_{1} \times V_{k}$.

The construction of the canonical decomposition tree of a bipartite Star $_{123}$-free graph can be obtained in linear time from the algorithm given by Quaddoura in [14]. According to this algorithm, every child of a $N$-node is a node marked by $P^{\prime}$ corresponding to a set $V_{i}, i=1, \cdots, k$, if $\left|V_{i}\right|>1$, or to a vertex of $G$ otherwise. Figure 3 illustrates a bipartite $\operatorname{Star}_{123}$-free graph and its canonical decomposition tree.

\section{Maximum Matching of Bipartite Star 123 -Free Graphs}

In this section we will extend the techniques developed in [2] to provide an $O(n)$ time algorithm for the maximum matching problem on bipartite Star $_{123}$-free graph. We present first the required tools for this purpose.

A classical tool for solving the maximum matching problem was introduced by Berge in [16]. Let $M$ be any matching of a graph $G=(V, E)$, an $M$-alternating path is a path whose edges are alternately in $M$ and in $E-M$. If some edge of $M$ is incident to a vertex $v$, this vertex is said to be saturated by $M$, otherwise $v$ is $M$-unsaturated. An $M$-augmenting path is an $M$-alternating path whose both endpoints are $M$-unsaturated.
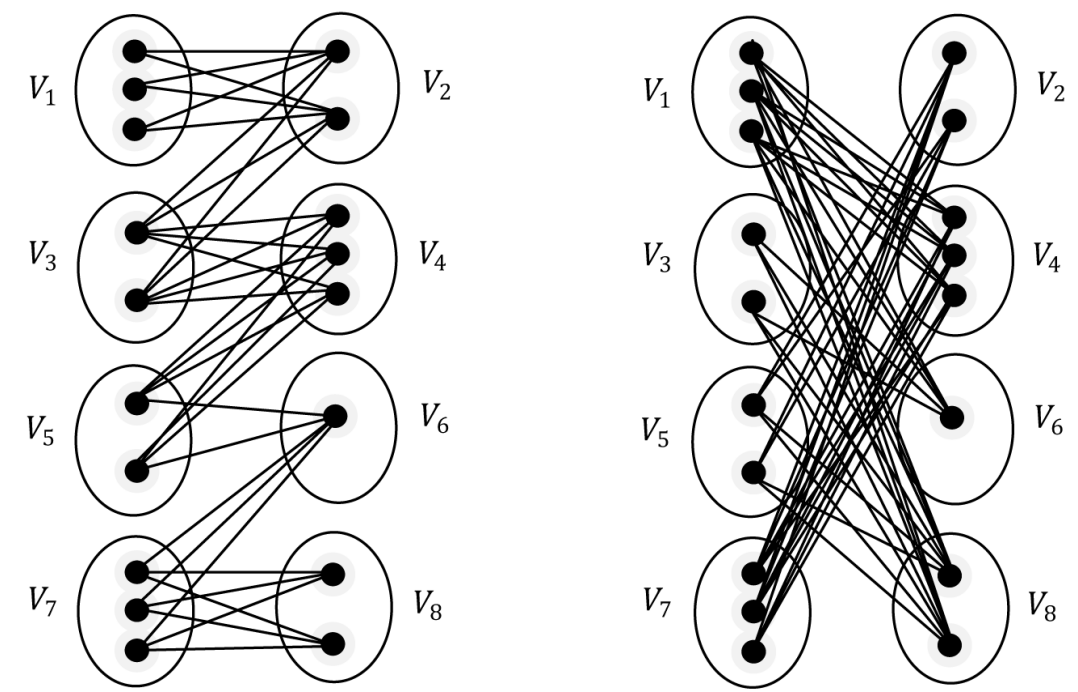

Figure 2. An $E P_{8}$ and an $\overline{E P}_{8}^{\text {bip }}$. 


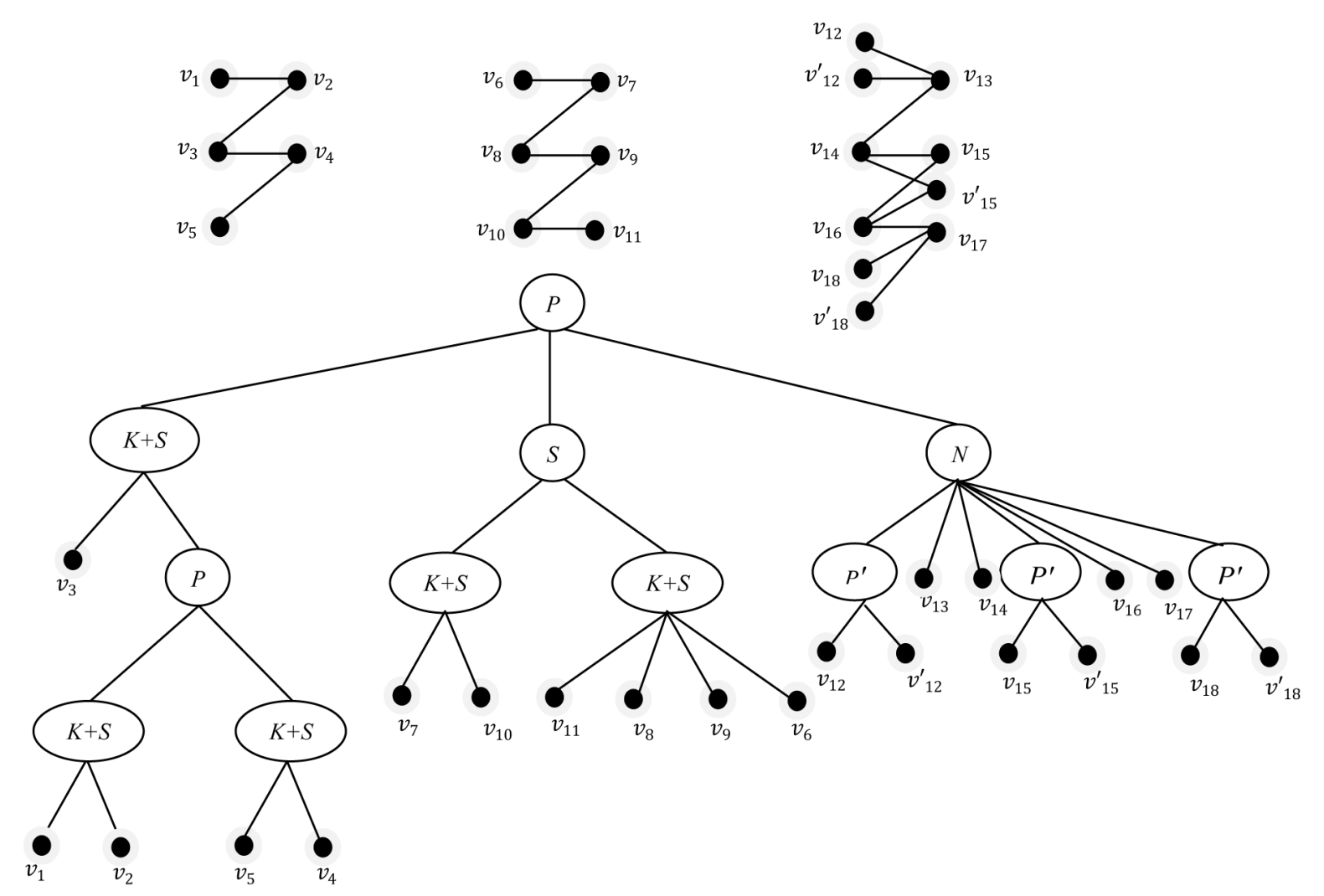

Figure 3. A bipartite Star $_{123}$-free graph and its canonical decomposition tree.

Theorem 7 [16]. A matching $M$ of a graph $G$ is maximum matching if and only if $G$ contain no M-augmenting path.

Consider a bipartite graph $G$ such that $G$ admit a decomposition according to some rule into two graphs $G_{1}$ and $G_{2}$. Let $M_{1}$ and $M_{2}$ be maximum matchings of $G_{1}$ and $G_{2}$, let $M=M_{1} \cup M_{2}$ which is a matching of $G$. In order to increase the size of $M$ we use the operations Match and Split (see [11]) described below.

Let $U_{1}$ be the set of $M_{1}$-unsaturated vertices of $G_{1}$ and $U_{2}$ be the set of $M_{2}$-unsatureted vertices of $G_{2}$. A Match operation occurs if there are two adjacent vertices $v_{1} \in U_{1}$ and $v_{2} \in U_{2}$ then the edge $v_{1} v_{2}$ is added to $M$, the vertices $v_{1}$ and $v_{2}$ are thus saturated by $M$ and they are respectively deleted from the sets $U_{1}$ and $U_{2}$.

Let $U$ be the set of $M$-unsaturated vertices, a Split operation occurs if there exists an edge of $M$ say $x y$ and vertices $u$ and $v$ belonging to $U$ such that $u$ is adjacent to $x$ and $v$ is adjacent to $y$. In that case the Split operation constructs a new matching $M^{\prime}$ defined by $M^{\prime}=M \bigcup\{u x, v y\}-\{x y\}$, the vertices $u$ and $v$ being saturated by $M^{\prime}$ and deleted from $U$ and the edge $x y$ is deleted from $M$. Note that, if $G=(B \cup W, E)$ is a bipartite complete then a maximum matching of $G$ can be obtained by applying Match operations between the two sets $B$ and $W$.

Let now $G$ be a bipartite $\operatorname{Star}_{123}$-free graph and $T(G)$ is its canonical decomposition tree. For our purpose we shall modify $T(G)$ to a binary tree $B T(G)$ as follows: We visit all nodes of $T(G)$ in DFS order. For a node $\alpha$ of type $P, S$ or $K+S$ let $\alpha_{1}, \cdots, \alpha_{k}$ be the children of $\alpha$. If $k=2$ then $\alpha$ does not change. Else $\alpha_{1}$ remains its left child and $\dot{\alpha}$ is its new child labeled by $P, S$ or $K+S$ respectively with $\alpha_{2}, \cdots, \alpha_{k}$ are its children. For a $N$-node $\alpha$, using the Procedure MAXMATCH $E P_{k}, E C_{k}$, the Procedure MAXMATCH $\overline{E P}_{k}^{b i p}$, or the procedure MAXMATCH $\overline{E C}_{k}^{\text {bip }}$ described below, which find a maximum matching of an extended path $E P_{k}$ or an extended cycle $E C_{k}$ or their bi-complements, we replace $\alpha$ by a leaf $\dot{\alpha}$ together with a maximum matching of the subgraph $G[\alpha]$ and the set of unsatureted vertices with respect to this matching. Our algorithm uses post order traversal to visit all the nodes of $B T(G)$. Whenever an internal node $\alpha$ of this binary tree is visited, we compute a maximum matching of $G[\alpha]$ from the maximum matching $M_{1}$ 
of $G\left[\alpha_{1}\right]$ and $M_{2}$ of $G\left[\alpha_{2}\right]$ where $\alpha_{1}$ and $\alpha_{2}$ are the two children of $\alpha$ in $B T(G)$. For this purpose we distinguish the following cases according to the type of $\alpha$.

\section{1. $\alpha$ Is of Type $P, S$ or $K+S$}

Consider now the set $M=M_{1} \cup M_{2}$ which is a matching of $G[\alpha]$. Obviously, if $\alpha$ is a $P$-node then $M$ is a maximum matching of $G[\alpha]$. In the case when $\alpha$ is of type $S$ or $K+S$ we use the same technique used in [6] to find a maximum matching of $G[\alpha]$.

Let $U_{1}$ be the set of $M_{1}$-unsaturated vertices of $G\left[\alpha_{1}\right]$ and $U_{2}$ be the set of $M_{2}$-unsatureted vertices of $G\left[\alpha_{2}\right]$. Let Match $\left[U_{1}, U_{2}\right]$ be the matching of $G[\alpha]$ obtained when all possible Match operations have been sequentially performed.

Let now $U$ be the set of $M$-unsaturated vertices, and Let Split $[U, M]$ be the matching obtained when all possible Split operations have been sequentially performed.

Theorem 8 [2]. If $\alpha$ is a $K+S$-node, the set Match $\left[U_{1}, U_{2}\right]$ is a maximum matching of $G[\alpha]$.

Theorem 9 [2]. Assume that $\alpha$ is a S-node and $M$ is equal to Match $\left[U_{1}, U_{2}\right]$. Let $U$ be the set of $M-u n-$ saturated vertices of $G[\alpha]$, then the set Split $[U, M]$ is a maximum matching of $G[\alpha]$.

\section{2. $\alpha$ Is of Type $N$}

In this section we will develop an $O(n)$ algorithm to find a maximum matching of an extended path $E P_{k}$ or an extended cycle $E C_{k}$ and an $O(n)$ algorithm to find a maximum matching of their bi-complement (see Definitions 5 and 6). We can suppose that $k=2 n^{\prime}$ if $k$ is even or $k=2 n^{\prime}+1$ if $k$ is odd. We denote by $\operatorname{Match}\left[V_{i}, V_{j}\right]$ the matching of the bipartite complete graph $G\left[V_{i} \cup V_{j}\right]$ obtained by Match operations between the two monochromatic sets $V_{i}$ and $V_{j}$. When an edge $x y$ is added to this matching where $x \in V_{i}$ and $y \in V_{j}$ then $x$ will be deleted from $V_{i}$ and $y$ will be deleted from $V_{j}$. Note that, during the execution of Procedure MAXMATCH $E P_{k}, E C_{k}$ or the Procedures MAXMATCH $\overline{E P_{k}^{\text {bip }}}$ and MAXMATCH $\overline{E C_{k}^{b i p}}$, the matching Match $\left[V_{i}, V_{j}\right]$ is not necessarily maximum for $G\left[V_{i} \cup V_{j}\right]$, this is because some vertices of $V_{i}$ or $V_{j}$ may already be saturated.

\subsection{1. $\alpha$ Is $E P_{k}$ or $E C_{k}$}

Procedure MAXMATCH $E P_{k}, E C_{k}$ provides a maximum matching of an extended path $E P_{k}$ or an extended cycle $E C_{k}$. By convention, every monochromatic set of an extended path or an extended cycle has an odd index consists of black vertices and those having an even index consist of white ones. For the purpose of simplification, the length $k$ of the extended path in this Procedure is considered to be odd, if this length is even then the set $V_{2 n^{\prime}+1}$ is considered to be empty.

\section{Procedure MAXMATCH $E P_{k}, E C_{k}$}

1) $M \leftarrow \varnothing$

2) if $G=E C_{k}$ then $M \leftarrow M \cup \operatorname{Match}\left[V_{1}, V_{k}\right]$

3) for $i=1$ to $n^{\prime}$ do

begin for

4) $\quad M \leftarrow M \cup \operatorname{Match}\left[V_{2 i-1}, V_{2 i}\right]$

5) $\quad M \leftarrow M \cup \operatorname{Match}\left[V_{2 i}, V_{2 i+1}\right]$

end for

Theorem 10. Let $G=(V, E)$ be an extended path $E P_{k}$ or an extended cycle $E C_{k}$ where

$V=V_{1} \cup V_{2} \cup \cdots \cup V_{2 n^{\prime}+1}$. Procedure MAXMATCH $E P_{k}, E C_{k}$ produces a maximum matching of $G$.

Proof. Let $P=v_{1} v_{2} \cdots v_{t}$ be an $M$-augmenting path in $G$. Since $G$ is a bipartite, $t$ is even, so $v_{1}$ and $v_{t}$ are of different colors. Without loss of generality, assume that $v_{1}$ is a black vertex and $v_{t}$ is white. Let $v_{1} \in V_{2 j-1}\left(1 \leq j \leq n^{\prime}+1\right)$ and $v_{t} \in V_{2 r}\left(1 \leq r \leq n^{\prime}\right)$.

Claim 1. There is no black vertex of $P$ in $V_{2 j+1}$.

Proof. Let $v_{s}$ be the first black vertex of $P$ in $V_{2 j+1}$, then $v_{s-1}$ must be in $V_{2 j}$. Since $v_{1}$ is a black vertex non saturated, it must be $v_{s-1} v_{s} \in M$. According to our Procedure, the edge $v_{s-1} v_{s}$ has been added to $M$ by the operation Match $\left[V_{2 j}, V_{2 j+1}\right]$, but before this step, the edge $v_{1} v_{s-1}$ must be added to $M$ by the step 
$\operatorname{Match}\left[V_{2 j-1}, V_{2 j}\right]$, a contradiction.

Claim 2. There is no white vertex of $P$ in $V_{2 r+2}$.

Proof. Let $v_{s}$ be the last white vertex of $P$ in $V_{2 r+2}$, then $v_{s+1}$ must be in $V_{2 r+1}$ or in $V_{2 r+3}$ or in $V_{1}$ and $v_{s} v_{s+1} \in M$. The vertex $v_{s+1}$ does not belong to $V_{2 r+3}$, otherwise, since $v_{t} \in V_{2 r}$, the path $v_{s+1} \cdots v_{t}$ must contain a vertex in $V_{2 r+2}$, a contradiction with our choice of $v_{s}$. If $v_{s+1} \in V_{2 r+1}$ then according to our Procedure, the edge $v_{s} v_{s+1}$ has been added to $M$ by the operation Match $\left[V_{2 r+1}, V_{2 r+2}\right]$ when $i=r+1$, but in the step $i=r$, the edge $v_{t} v_{s+1}$ must be added to $M$ by the operation Match $\left[V_{2 r}, V_{2 r+1}\right]$, a contradiction. If $v_{s+1} \in V_{1}$ then $G=E C_{k}$ and $2 r+2=2 n^{\prime}$. In this case, the vertex $v_{1}$ does not belong to $V_{2 n^{\prime}-1}$ or to $V_{2 n^{\prime}-3}$, otherwise $v_{1} v_{t}$ must be added to $M$, so $j<n^{\prime}-2$. But now the set $V_{2 j+1}$ must contain a vertex of $P$, a contradiction with Claim 1.

Suppose that $G$ is an $E P_{k}$ or $G$ is an $E C_{k}$ such that there is no edge of $P$ connecting $V_{1}$ and $V_{k}$. If $j<r$ then either $V_{2 j+1}$ contains a vertex of $P$ or there is an edge of $P$ connecting $V_{1}$ and $V_{k}$, a contradiction. If $j>r$ then either $V_{2 r+2}$ contains a vertex of $P$ or there is an edge of $P$ connecting $V_{1}$ and $V_{k}$, a contradiction. Therefore $j=r$. But now the edge $v_{1} v_{t}$ must be added to $M$ by the operation Match $\left[V_{2 r-1}, V_{2 r}\right]$, a contradiction.

Suppose now $G$ is an $E C_{k}$ and there is an edge of $P$ connecting $V_{1}$ and $V_{k}$. Let $v_{i} V_{i+1}$ be the first edge of $P$ connecting $V_{1}$ and $V_{k}$. By Claim 1 and Claim 2, $v_{t} \in V_{k}$. Thus $v_{1}$ does not belong to $V_{1} \cup V_{k-1}$. If $v_{i} \in V_{k}$ then according to our choice of $v_{i} v_{i+1}$, the set $V_{2 j+1}$ must contain a vertex of $P$, a contradiction with Claim 1 . Therefore $v_{i} \in V_{1}$. Since the vertex $v_{1}$ is a black non-saturated vertex, the edge $v_{i} v_{i+1}$ does not belong to $M$, By our choice of the edge $v_{i} v_{i+1}$, the vertex $v_{i-1}$ must belong to $V_{2}$. Now, the edge $v_{i-1} v_{i}$ has been added to $M$ by the operation Match $\left[V_{1}, V_{2}\right]$. But before this step, the edge $v_{i} v_{t}$ must be added to $M$ by the operation $\operatorname{Match}\left[V_{1}, V_{k}\right]$, a contradiction. $\square$

The following Table 1 illustrates a trace of the Procedure MAXMATCH $E P_{k}, E C_{k}$ for the $E P_{8}$ in Figure 2 where a vertex in $V_{i}$ is denoted by $v_{i}^{j}, i=1, \cdots, 8, j=1, \cdots,\left|V_{i}\right|$.

\subsection{2. $\alpha$ Is $\overline{E P}_{k}^{b i p}$ or $\overline{E C}_{k}^{b i p}$}

Note that the matching obtained by the Procedure MAXMATCH $E P_{k}, E C_{k}$ is ensured to be maximum because of the order of applying Match operations. In the case when $\alpha$ is $\overline{E P}{ }_{k}^{b i k}$ or $\overline{E C}_{k}^{b i p}$, we must also design an order of applying Match operations and Split operations to ensure that the resulting matching is maximum. For this purpose, we will study first the structure of a $M$-augmenting path of a matching $M$ of $\alpha$ obtained by doing in an arbitrary order all possible Match operations then all possible Split operations (Lemma 12 and Lemma 13). Knowing this structure will enable us to design an order of applying Match operations (Procedure Match $(G)$ )

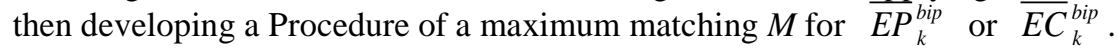

Recall that when an edge $x y$ is added to a matching $M$ by a Match operation $\operatorname{Match}\left[V_{i}, V_{j}\right]$ where $x \in V_{i}$ and $y \in V_{j}$ then $x$ will be deleted from $V_{i}$ and $y$ will be deleted from $V_{j}$. In addition, we suppose here that Match operation associates labels with $x$ and $y$ as $\operatorname{Label}(x)=i$ and $\operatorname{Label}(y)=j$ when $x \in V_{i}$ and $y \in V_{j}$ respectively. Two monochromatic sets $V_{i}$ and $V_{j}$ of different color are called independent if $V_{i} \cup V_{j}$ form a stable set, non-independent otherwise.

Lemma 11. Let $G=\overline{E P}_{k}^{b i p}$ or $G=\overline{E C}_{k}^{\text {bip }}$, let $M$ be a matching of $G$ obtained when all possible Match operations have been performed. If there are at least two $M$-unsaturated vertices of different color then all the $M$-unsaturated vertices are located in at most three consecutive monochromatic sets $V_{s}, V_{s+1}$ and $V_{s+2}$ where $s \in\{1, \cdots, k\}$.

\begin{tabular}{cccc}
\multicolumn{2}{c}{ Table 1. Illustration of procedure MAXMATCH } & $E P_{k}, E C_{k} S$ for the $E P_{8}$ in Figure 2. \\
\hline$i$ & Match $\left[V_{2 i-1}, V_{2 i}\right]$ & Match $\left[V_{2 i}, V_{2 i+1}\right]$ & $M$ \\
\hline 1 & $\left\{v_{1}^{1} v_{2}^{1}, v_{1}^{2} v_{2}^{2}\right\}$ & - & $\left\{v_{1}^{1} v_{1}^{2}, v_{2}^{1} v_{2}^{2}\right\}$ \\
2 & $\left\{v_{3}^{1} v_{4}^{1}, v_{3}^{2} v_{4}^{2}\right\}$ & $\left\{v_{4}^{3} v_{5}^{1}\right\}$ & $\left\{v_{1}^{1} v_{1}^{2}, v_{2}^{1} v_{2}^{2}, v_{3}^{1} v_{4}^{1}, v_{3}^{2} v_{4}^{2}, v_{4}^{3} v_{5}^{1}\right\}$ \\
3 & $\left\{v_{5}^{2} v_{6}^{1}\right\}$ & - & $\left\{v_{1}^{1} v_{1}^{2}, v_{2}^{1} v_{2}^{2}, v_{3}^{1} v_{4}^{1}, v_{3}^{2} v_{4}^{2}, v_{4}^{3} v_{5}^{1}, v_{5}^{2} v_{6}^{1}\right\}$ \\
4 & $\left\{v_{7}^{1} v_{8}^{1}, v_{7}^{2} v_{8}^{2}\right\}$ & - & $\left\{v_{1}^{1} v_{1}^{2}, v_{2}^{1} v_{2}^{2}, v_{3}^{1} v_{4}^{1}, v_{3}^{2} v_{4}^{2}, v_{4}^{3} v_{5}^{1}, v_{5}^{2} v_{6}^{1} v_{7}^{1} v_{8}^{1}, v_{7}^{2} v_{8}^{2}\right\}$ \\
\hline
\end{tabular}


Proof. By the hypothesis of the Lemma, all the $M$-unsaturated vertices must be in independent sets. Obviously any three consecutive sets are independent and the maximum number of independent sets is three.

Assume that $G=\overline{E P}_{k}^{\text {bip }}$ or $G=\overline{E C}_{k}^{\text {bip }}$ and $M$ is a matching of $G$ obtained when all possible Match operations have been performed (in an arbitrary order). The following Procedure determines the sets $V_{s}, V_{s+1}, V_{s+2}$ which are the possible location of $M$-unsaturated vertices. Note that when $G=\overline{E C}_{k}^{b i p}$, the sets $V_{k}$ and $V_{1}$ are consecutive.

\section{Procedure $M$-unsaturated vertices $(G, M)$}

1) Find the small index $s \in\{1, \cdots, k\}$ for which $V_{s} \neq \varnothing$

2) if there is no such $s$ then return $M$ is maximum else

3) if $G=\overline{E P}_{k}^{b i p}$ or $s \neq 1$ then

//when $G=\overline{E P}_{k}^{b i p}$ and $s=k-1, V_{s+2}$ does not exist, when $G=\overline{E C}_{k}^{b i p}$ and $s=k-1, V_{s+2}=\varnothing$

4) if $s=k-1$ then return $V_{s}=V_{k-1}$ and $V_{s+1}=V_{k}$

5) else return $V_{s}, V_{s+1}, V_{s+2}$ else $/ / G=\overline{E C}_{k}^{\text {bip }}$ and $s=1$

6) if $V_{k}=\varnothing$ then return $V_{s}=V_{1}, V_{s+1}=V_{2}, V_{s+2}=V_{3}$

7) else if $V_{k-1}=\varnothing$ then return $V_{s}=V_{k}, V_{s+1}=V_{1}, V_{s+2}=V_{2}$

8) else return $V_{s}=V_{k-1}, V_{s+1}=V_{k}, V_{s+2}=V_{1}$

According to Lemma 11, one of the two $M$-unsaturated vertices of any $M$-augmenting path in $G$ is in $V_{s}$ and the second in $V_{s+1}$, or one in $V_{s+1}$ and the second in $V_{s+2}$. Consider first a $M$-augmenting path in $G$ which its $M$-unsaturated vertices are in $V_{s}$ and in $V_{s+1}$.

To augment the size of $M$, Split operations can be done between the $M$-unsaturated vertices of $V_{s}$, the $M$-unsaturated vertices of $V_{s+1}$ and the edges of $M$ whose extremities belong to monochromatic sets non-independent of $V_{s}$ and $V_{s+1}$, namely the edges of $M$ whose extremities don't belong to $V_{s-1}, V_{s}, V_{s+1}$ when $G=\overline{E P}_{k}^{b i p}$ and $s=k-1$, and the edges of $M$ whose extremities don't belong to $V_{s-1}, V_{s}, V_{s+1}$ and $V_{s+2}$ otherwise. The following Procedure performs these Split operations.

\section{Procedure Split $\left(M, V_{s}, V_{s+1}\right)$}

1) if $G=\overline{E P}_{k}^{b i p}$ and $s=k-1$ then

$$
M^{\prime}=\{x y \in M \mid \operatorname{label}(x) \text { and } \operatorname{label}(y) \neq s-1, s \text { and } s+1\}
$$

2) else $M^{\prime}=\{x y \in M \mid \operatorname{label}(x)$ and $\operatorname{label}(y) \neq s-1, s, s+1$ and $s+2\}$

3) while $V_{s} \neq \varnothing$ and $V_{s+1} \neq \varnothing$ and $M^{\prime} \neq \varnothing$ do

Begin while

4) let $u \in V_{s}, v \in V_{s+1}, x y \in M^{\prime}, M=M-\{x y\} \cup\{u x, v y\}$

5) $\quad V_{s}=V_{s}-\{u\}, V_{s+1}=V_{s+1}-\{v\}, M^{\prime}=M^{\prime}-\{x y\}$

$/ /$ assuming that $u$ and $x$ also $v$ and $y$ are of different color

end while

The following Lemma describes the structure of a $M$-augmenting path whose extremities belong to $V_{s}$ and $V_{s+1}$.

Lemma 12. After the execution of Procedure Split $\left(M, V_{s}, V_{s+1}\right)$ if there is a M-augmenting path $P=v_{1} \cdots v_{t}$ in $G$ whose extremities in $V_{s}$ and $V_{s+1}$ then:

- $G \neq \overline{E P}_{k}^{b i p}$ or $s \neq k-1$.

- $P$ can be reduced to a M-augmenting path $v_{1} v_{2} v_{3} v_{4} v_{5} v_{6}$ where $v_{1} \in V_{s}, v_{2} \in V_{i}, v_{3} \in V_{s+2}, v_{4} \in V_{s-1}, v_{5} \in V_{j}$, $v_{6}=v_{t} \in V_{s+1}, V_{i}$ is any non-independent set of $V_{s}$ and $V_{j}$ is any non-independent set of $V_{s+1}$.

Proof. Let $P=v_{1} v_{2} \cdots v_{t}$ be a $M$-augmenting path in $G$ where $v_{1} \in V_{s}$ and $v_{t} \in V_{s+1}$. Since after the execution of Procedure Split $\left(M, V_{s}, V_{s+1}\right), V_{s} \neq \varnothing$ and $V_{s+1} \neq \varnothing$, the set $M^{\prime}$ must be empty. Therefore, if $G=\overline{E P}_{k}^{\text {bip }}$ and $s=k-1$, every edge of $M$ has an extremity in $V_{s-1}, V_{s}$ or $V_{s+1}$, and if $G \neq \overline{E P}_{k}^{\text {bip }}$ or $s \neq k-1$, every edge of $M$ has an extremity in $V_{s-1}, V_{s}, V_{s+1}$ or $V_{s+2}$. Obviously, the color of every vertex of $P$ having an odd index (resp. even index) is as the color of $v_{1}$ (resp. $v_{t}$ ).

Let $v_{i}$ be the first vertex of $P$ having an odd index and belongs to a set distinct of $V_{s}$, then $v_{i-1} v_{i} \in M$. 
Since $M^{\prime}=\varnothing$ and $v_{i} \notin V_{s}$, either $v_{i-1} \in V_{s-1} \cup V_{s+1}$ or $v_{i} \in V_{s+2}$. If $v_{i-1} \in V_{s-1} \cup V_{s+1}$ then $v_{i-2} \notin V_{s}$, a contradiction with our choice of $v_{i}$, therefore $v_{i} \in V_{s+2}$. Since $V_{s+2}$ does not exist when $G=\overrightarrow{E P}_{k}^{b i p}$ and $s=k-1$, then $G \neq \overline{E P}_{k}^{b i p}$ or $s \neq k-1$. Since $v_{i-1} \notin V_{s-1} \cup V_{s+1}, v_{1} v_{i-1} \in E(G)$. Now the subpath $v_{1} v_{2} \cdots v_{i}$ of $P$ can be reduced to $v_{1} v_{i-1} v_{i}$.

Let $v_{j}$ be the last vertex of $P$ having an even index and belongs to a set distinct of $V_{s+1}$, then $v_{j} v_{j+1} \in M$. Since $M^{\prime}=\varnothing$ and $v_{j} \notin V_{s+1}$, either $v_{j+1} \in V_{s} \cup V_{s+2}$ or $v_{j} \in V_{s-1}$. If $v_{j+1} \in V_{s} \cup V_{s+2}$ then $v_{j+2} \notin V_{s+1}$, a contradiction with our choice of $v_{j}$, therefore $v_{j} \in V_{s-1}$. Since $v_{i} \in V_{s+2}$ and $v_{j} \in V_{s-1}, v_{i} v_{j} \in E(G)$. Obviously $v_{i} v_{j} \notin M$. Since $v_{j+1} \notin V_{s} \cup V_{s+2}, \quad v_{j+1} v_{t} \in E(G)$. Now the path $P$ can be reduced to the $M$-augmenting path $v_{1} v_{i-1} v_{i} v_{j} v_{j+1} v_{t}$.

Consider now a $M$-augmenting path in $G$ such that its $M$-unsaturated vertices are in $V_{s+1}$ and in $V_{s+2}$. In a similar way, by replacing in the above Procedure and in Lemma 12, $s-1$ by $s, s$ by $s+1, s+1$ by $s+2$ and $s+2$ by $s+3$, we obtain the Procedure Split $\left(M, V_{s+1}, V_{s+2}\right)$ and Lemma 13which describes the structure of a $M$-augmenting path whose extremities belong to $V_{s+1}$ and $V_{s+2}$.

Procedure Split $\left(M, V_{s+1}, V_{s+2}\right)$

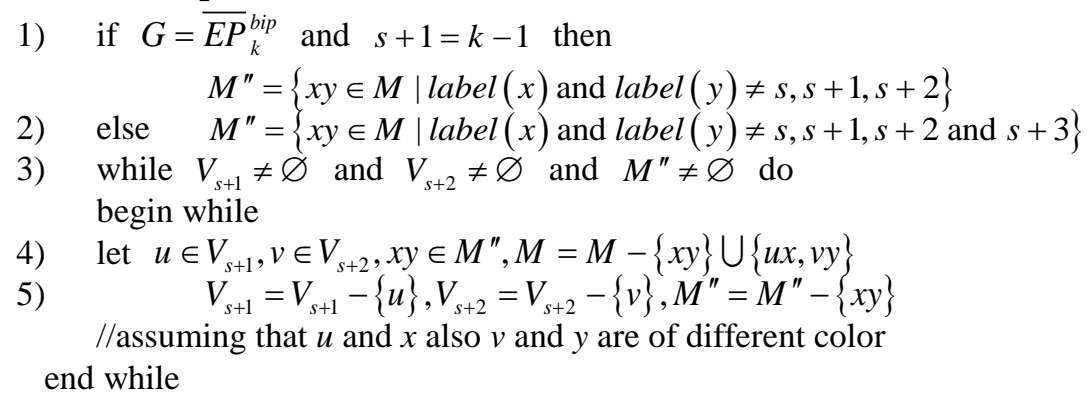

Lemma 13. After the execution of Procedure Split $\left(M, V_{s+1}, V_{s+2}\right)$ if there is a M-augmenting path $P=v_{1} \cdots v_{t}$ in $G$ whose extremities in $V_{s+1}$ and $V_{s+2}$ then:

- $G \neq \overline{E P}_{k}^{\text {bip }}$ or $s+1 \neq k-1$.

- $P$ can be reduced to a M-augmenting path $v_{1} v_{2} v_{3} v_{4} v_{5} v_{6}$ where $v_{1} \in V_{s+1}, v_{2} \in V_{i}, v_{3} \in V_{s+3}, v_{4} \in V_{s}, v_{5} \in V_{j}$, $v_{6}=v_{t} \in V_{s+2}, V_{i}$ is any non-independent set of $V_{s+1}$ and $V_{j}$ is any non-independent set of $V_{s+2}$.

We start now by developing a Procedure for a maximum matching in $G=\overline{E P}_{k}^{\text {bip }}$ or $G=\overline{E C}_{k}^{\text {bip }}$. The order of applying Match operations is defined in following Procedure which called MATCH $(G)$. Recall that either $k=2 n^{\prime}$ or $k=2 n^{\prime}+1$.

\section{Procedure MATCH (G)}

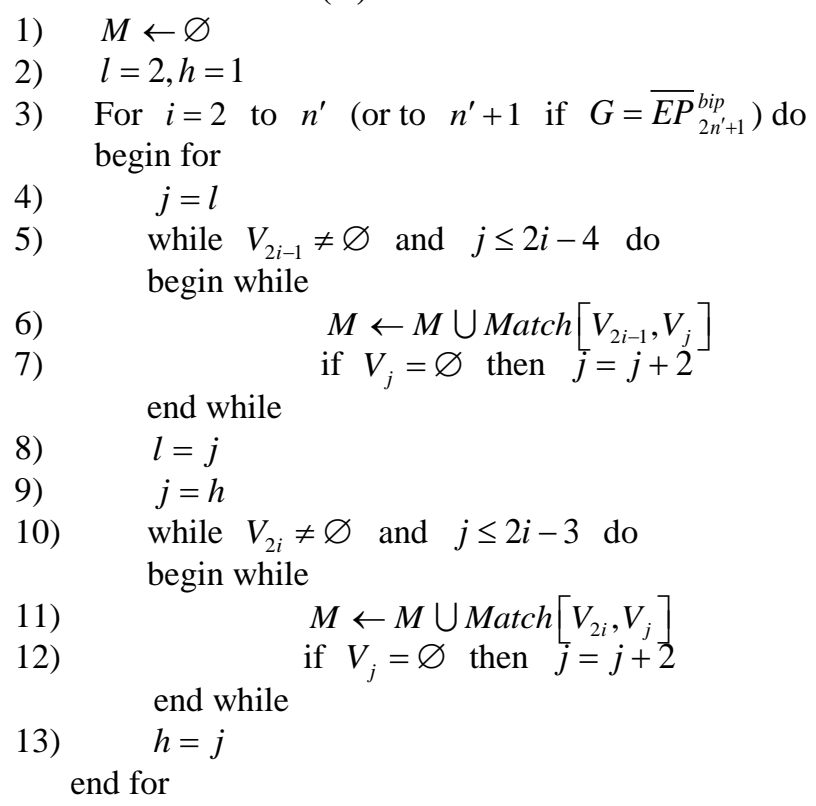


Procedure MATCH $(G)$ works as following, for every $i=2$ to $n^{\prime}$ (or to $n^{\prime}+1$ if $G=\overline{E P}_{2 n^{\prime}+1}^{b i p}$ ):

- Add to $M$ the possible edges between $V_{2 i-1}$ as long as it is non empty and (the non-independent sets of $V_{2 i-1}$ having indices less than 2i-1) $V_{l}, V_{l+2}, \cdots, V_{2 i-4}$ with respect to this order, where $l$ determines the last non empty set in $V_{2}, \cdots, V_{2 i-2}$ during the for loop iterations $2, \cdots, i-1$.

- Add to $M$ the possible edges between $V_{2 i}$ as long as it is non empty and (the non-independent sets of $V_{2 i}$ having indices less than 2i) $V_{h}, V_{h+2}, \cdots, V_{2 i-3}$ with respect to this order, where $h$ determines the last non empty set in $V_{1}, \cdots, V_{2 i-1}$ during the for loop iterations $2, \cdots, i-1$.

Observation 14. According to Procedure MATCH $(G)$ :

- if $x y$ is an edge of $M$ created by the operation $\operatorname{Match}\left[V_{i}, V_{j}\right]$ then $i>j$.

- if $i<i^{\prime}$ (resp. $j<j^{\prime}$ ) then the edges of $M a t c h\left[V_{i}, V_{j}\right]$ have been added to $M$ before adding the edges of $\operatorname{Match}\left[V_{i^{\prime}}, V_{j}\right]$ (resp. Match $\left[V_{i}, V_{j^{\prime}}\right]$ ).

- if $i>j>r$ then the edges of Match $\left[V_{j}, V_{r}\right]$ have been added to $M$ before adding the edges of $\operatorname{Match}\left[V_{i}, V_{j}\right]$.

The following Table 2 illustrates a trace of the Procedure MATCH $(G)$ for the $\overline{E P}_{8}^{b i p}$ in Figure 2 . The second and the third column of this table represent the execution of steps 5 and 10 respectively.

The combination of Procedures MATCH $(G), M$-unsaturated vertices $(G, M)$, Split $\left(M, V_{s}, V_{s+1}\right)$, and Split $\left(M, V_{s+1}, V_{s+2}\right)$ provides the Procedure MAXMMATCH $\overline{E P}_{k}^{\text {bip }}$. For a maximum matching of $\overline{E C}_{k}^{\text {bip }}$ we need a little addition. Theorem 15 proves their correctness.

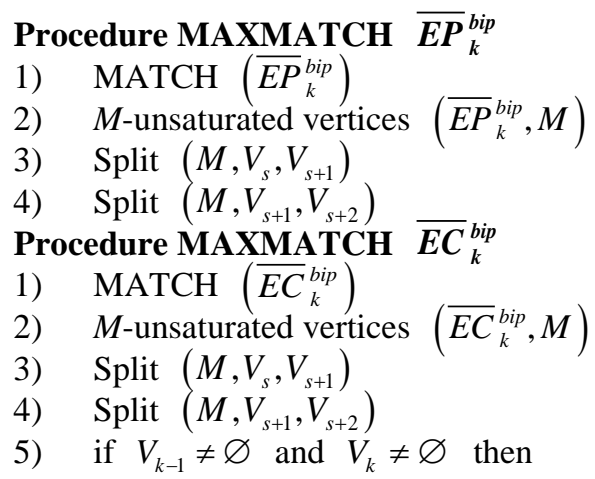

5) if $V_{k-1} \neq \varnothing$ and $V_{k} \neq \varnothing$ then

//Assuming that $x$ and the vertices of $V_{k}$ also $y$ and the vertices of $V_{k-1}$ are of the same color

6) $\quad M^{\prime}=\{x y \in M \mid \operatorname{label}(x) \neq k-2$, $\operatorname{label}(y)=1\} \neq \varnothing$

7) $\quad M^{\prime \prime}=\{x y \in M \mid \operatorname{label}(x)=k-2$, label $(y) \neq 1\}$

8) while $V_{k-1} \neq \varnothing$ and $V_{k} \neq \varnothing$ and $M^{\prime} \neq \varnothing$ and $M^{\prime \prime} \neq \varnothing$ do begin while

$$
\text { let } u \in V_{k-1}, v \in V_{k}, x y \in M^{\prime}, x^{\prime} y^{\prime} \in M^{\prime \prime}
$$

9) $\quad M \leftarrow M-\left\{x y, x^{\prime} y^{\prime}\right\} \bigcup\left\{u x, x^{\prime} y, v y^{\prime}\right\}$

10) $\quad V_{k-1}=V_{k-1}-\{u\}, V_{k}=V_{k}-\{v\}, M^{\prime}=M^{\prime}-\{x y\}, M^{\prime \prime}=M^{\prime \prime}-\left\{x^{\prime} y^{\prime}\right\}$

end while

Theorem 15. Procedure MAXMATCH $\overline{E P}_{k}^{b i p}$ and Procedure MAXMATCH $\overline{E C}_{k}^{\text {bip }}$ produce a maximum matching of $G=\overline{E P}_{k}^{\text {bip }}$ and $G=\overline{E C}_{k}^{b i p}$ respectively.

Proof. Suppose that after execution of Procedure MAXMATCH $\overline{E P}_{k}^{\text {bip }}$ or Procedure MAXMATCH $\overline{E C}_{k}^{b i p}$ there is a $M$-augmenting path $P=v_{1} v_{2} \cdots v_{t}$. Since $v_{1}$ and $v_{t}$ are of different color and all the monochromatic sets are empty except at most $V_{s}, V_{s+1}$ and $V_{s+2}$, there are two cases, either $v_{1} \in V_{s}, v_{t} \in V_{s+1}$ or $v_{1} \in V_{s+1}, v_{t} \in V_{s+2}$.

Let $r=s$ when $v_{1} \in V_{s}, v_{t} \in V_{s+1}$ or $r=s+1$ when $v_{1} \in V_{s+1}, v_{t} \in V_{s+2}$. By Lemma 12 and Lemma 13, $P$ can be reduced to a path $v_{1} v_{2} v_{3} v_{4} v_{5} v_{6}$ where $v_{1} \in V_{r}, v_{2} \in V_{i}, v_{3} \in V_{r+2}, v_{4} \in V_{r-1}, v_{5} \in V_{j}, v_{6}=v_{t} \in V_{r+1}, V_{i}$ is any non-independent set of $V_{r}$ and $V_{j}$ is any non-independent set of $V_{r+1}$. Assume first that $r \neq k-1$ and $r \neq k$.

Claim 1. The edge $v_{2} v_{3}$ is obtained by Split operation.

Proof. Suppose that the edge $v_{2} v_{3}$ is obtained by Match operation. Since $v_{3} \in V_{r+2}$ the edge $v_{2} v_{3}$ is obtained either by $\operatorname{Match}\left[V_{r+2}, V_{i}\right]$ or by $\operatorname{Match}\left[V_{i}, V_{r+2}\right]$. Without loss of generality, assume that $v_{2} v_{3}$ is ob- 
Table 2. Illustration of Procedure MATCH $(\mathrm{G})$ for the $\overline{E P}_{8}^{\text {bip }}$ in Figure 2.

\begin{tabular}{cccccccc}
\hline$i$ & $h$ & $l$ & $j$ & $\operatorname{Match}\left[V_{2 i-1}, V_{j}\right]$ & $j$ & $\operatorname{Match}\left[V_{2 i}, V_{j}\right]$ & $M$ \\
\hline 2 & 2 & 2 & 2 & - & 1 & $\left\{v_{4}^{1} v_{1}^{1}, v_{4}^{2} v_{1}^{2}, v_{4}^{3} v_{1}^{3}\right\}$ & $\left\{v_{4}^{1} v_{1}^{1}, v_{4}^{2} v_{1}^{2}, v_{4}^{3} v_{1}^{3}\right\}$ \\
& & & 2 & - & 3 & - & \\
3 & 2 & 3 & 2 & $\left\{v_{5}^{1} v_{2}^{1}, v_{5}^{2} v_{2}^{2}\right\}$ & 3 & $\left\{v_{6}^{1} v_{3}^{1}\right\}$ & $\left\{v_{4}^{1} v_{1}^{1}, v_{4}^{2} v_{1}^{2}, v_{4}^{3} v_{1}^{3}, v_{5}^{1} v_{2}^{1}, v_{5}^{2} v_{2}^{2}, v_{6}^{1} v_{3}^{1}\right\}$ \\
& & & 4 & - & 3 & - & \\
4 & 4 & 3 & 4 & - & 3 & $\left\{v_{8}^{1} v_{3}^{2}\right\}$ & $\left\{v_{4}^{1} v_{1}^{1}, v_{4}^{2} v_{1}^{2}, v_{4}^{3} v_{1}^{3}, v_{5}^{1} v_{2}^{1}, v_{5}^{2} v_{2}^{2}, v_{1}^{6} v_{1}^{3}, v_{8}^{1} v_{3}^{2}\right\}$ \\
\hline
\end{tabular}

tained by Match $\left[V_{r+2}, V_{i}\right]$. By Observation 14, $r+2>i$. Since $v_{1} \in V_{r}, r>i$. So the operation $\operatorname{Match}\left[V_{r}, V_{i}\right]$ exists and must precedes the operation $\operatorname{Match}\left[V_{r+2}, V_{i}\right]$ by Observation 14 and since $r \neq k-1, k$. Therefore, the edge $v_{1} v_{2}$ must be added to $M$ instead of adding the edge $v_{2} v_{3}$, a contradiction.

Claim 2. $r=s$.

Proof. If $r=s+1$ then $v_{3} \in V_{s+3}$. By Claim $1, v_{2} v_{3}$ is obtained by Split operation, thus the vertex $v_{2}$ must belong to $V_{s+2}$, a contradiction since $V_{s+2}$ and $V_{s+3}$ are independent.

Since $v_{3} \in V_{s+2}$, the edge $v_{2} v_{3}$ is obtained by the step 4, that is by Split $\left(M, V_{s+1}, V_{s+2}\right)$. Let $x y \in M^{\prime \prime}$ be the edge which was in $M$ and which has been used in step 4 of the Procedure Split $\left(M, V_{s+1}, V_{s+2}\right)$ to obtain the edge $v_{2} v_{3}$. The vertex $v_{2}$ must be identical to $x$ or to $y$. Let $x$ be the vertex $v_{2}$. By the definition of $M^{\prime \prime}$, the vertices $x$ and $y$ don't belong to $V_{s} \cup V_{s+1} \cup V_{s+2}$. Since $v_{1} \in V_{s}$ and $v_{1} v_{2} \in E, \quad x=v_{2} \notin V_{s-1}$. Obviously, $y \notin V_{s-1}$. Therefore $x y \in M^{\prime}$ where $M^{\prime}$ is the set defined in step 4 of the Procedure Split $\left(M, V_{s}, V_{s+1}\right)$. But before executing the step Split $\left(M, V_{s+1}, V_{s+2}\right)$, the set $M^{\prime}$ must be empty since $V_{s} \neq \varnothing, V_{s+1} \neq \varnothing$, a contradiction.

Assume that $r=k-1$. Then $v_{1} \in V_{k-1}$ and $v_{t} \in V_{k}$. By Lemma 12 and Lemma 13, $G=\overline{E C}_{k}^{b i p}, v_{1} \in V_{k-1}$, $v_{2} \in V_{i}, v_{3} \in V_{1}, v_{4} \in V_{k-2}, v_{5} \in V_{j}, v_{6}=v_{t} \in V_{k}, V_{i}$ is any non-independent set of $V_{k-1}$ and $V_{j}$ is any non-independent set of $V_{k}$. Since $v_{1} \in V_{k-1}, v_{6} \in V_{k}$ then $V_{k-1} \neq \varnothing$ and $V_{k} \neq \varnothing$. Therefore one of the sets $M^{\prime}$ and $M^{\prime \prime}$ in step 8 must be empty. This is contradicted with the fact that $v_{2} v_{3} \in M^{\prime}$ and $v_{4} v_{5} \in M^{\prime \prime}$.

Assume finally that $r=k$. Then $v_{1} \in V_{k}$ and $v_{t} \in V_{1}$. Since in this case $V_{k}$ and $V_{1}$ must be independent, $G=\overline{E C}_{k}^{b i p}$. By Lemma 12 and Lemma 13, $v_{1} \in V_{k}, v_{2} \in V_{i}, v_{3} \in V_{2}, v_{4} \in V_{k-1}, v_{5} \in V_{j}, v_{6}=v_{t} \in V_{1}, V_{i}$ is any non-independent set of $V_{k}$ and $V_{j}$ is any non-independent set of $V_{1}$.

Claim 3. The edge $v_{4} v_{5}$ is obtained by Split operation.

Proof. Suppose that the edge $v_{4} v_{5}$ is obtained by Match operation. Since $k-1>j, v_{4} \in V_{k-1}$ and $v_{5} \in V_{j}$, then the edge $v_{4} v_{5}$ is obtained by $\operatorname{Match}\left[V_{k-1}, V_{j}\right]$. By Observation 14, since $k-1>j>1$, the operation $\operatorname{Match}\left[V_{j}, V_{1}\right]$ exists and must precede the operation Match $\left[V_{k-1}, V_{j}\right]$. Therefore, the edge $v_{5} v_{6}$ must be added to $M$ instead of adding the edge $v_{4} v_{5}$, a contradiction.

Claim 4. $r=s+1$.

Proof. If $r=s$ then $v_{4} \in V_{s-1}$. By Claim 3, $v_{4} v_{5}$ is obtained by Split operation, thus the vertex $v_{5}$ must belong to $V_{s}$, a contradiction since $V_{s-1}$ and $V_{s}$ are independent.

Since $v_{4} \in V_{s}$, the edge $v_{4} v_{5}$ is obtained by the step 3, that is by Split $\left(M, V_{s}, V_{s+1}\right)$. Let $x y \in M^{\prime}$ be the edge which was in $M$ and which has been used in step 4 of the Procedure Split $\left(M, V_{s}, V_{s+1}\right)$ to obtain the edge $v_{4} v_{5}$. The vertex $v_{5}$ must be identical to $x$ or to $y$. Let $x$ be the vertex $v_{5}$ and let $y \in V_{h}$. By the definition of $M^{\prime}, h \neq 1$. Obviously, $x y$ was not created by split operation. By Observation 14, if $j>h$ (resp. $j<h$ ) then $x y$ was created by $\operatorname{Match}\left[V_{j}, V_{h}\right]$ (resp. Match $\left[V_{h}, V_{j}\right]$ ). Since $1<h$ (resp. $h>j>1$ ), the operation $\operatorname{Match}\left[V_{j}, V_{1}\right]$ precedes the operation $\operatorname{Match}\left[V_{j}, V_{h}\right]$ (resp. Match $\left[V_{h}, V_{j}\right]$ ). So the edge $v_{5} v_{6}$ must be added to $M$ instead of adding $x y$, a contradiction. $\square$

Lets apply the Procedure MAXMATCH $\overline{E P}_{k}^{\text {bip }}$ on the graph $G=\overline{E P}_{8}^{b i p}$ in Figure 2. As we shown above, Procedure MATCH $(G)$ produces the matching $M=\left\{v_{4}^{1} v_{1}^{1}, v_{4}^{2} v_{1}^{2}, v_{4}^{3} v_{1}^{3}, v_{5}^{1} v_{2}^{1}, v_{5}^{2} v_{2}^{2}, v_{1}^{6} v_{1}^{3}, v_{8}^{1} v_{3}^{2}\right\}$. Procedure $M$-unsaturated vertices $(G, M)$ gives that $V_{s}=V_{k-1}=V_{7}, V_{s+1}=V_{k}=V_{8}$ and $V_{s+2}$ does not exist. Procedure Split $\left(M, V_{s}, V_{s+1}\right)$ gives that $M^{\prime}=\left\{v_{4}^{1} v_{1}^{1}, v_{4}^{2} v_{1}^{2}, v_{4}^{3} v_{1}^{3}, v_{5}^{1} v_{2}^{1}, v_{5}^{2} v_{2}^{2}\right\}$ and $M=\left\{v_{4}^{1} v_{1}^{1}, v_{4}^{2} v_{1}^{2}, v_{4}^{3} v_{1}^{3}, v_{5}^{1} v_{2}^{1}, v_{8}^{2} v_{5}^{2}, v_{7}^{1} v_{2}^{1}, v_{6}^{1} v_{3}^{1}, v_{8}^{1} v_{3}^{2}\right\}$. Since $V_{s+2}$ does not exist, Procedure Split $\left(M, V_{s+1}, V_{s+2}\right)$ gives nothing. 


\subsection{The Whole Algorithm}

Let us present now our algorithm for the maximum matching problem on bipartite Star $_{123}$-free graphs. Theorems 8, 9, 10, and 15 prove its correctness.

\section{Algorithm Maximum Matching}

Input: A bipartite $\operatorname{Star}_{123}$-free graph $G$ and its binary canonical decomposition tree $B T(G)$.

Output: $M$ a maximum matching of $G$ and $U$ the set of $M$-unsaturated vertices of $G$.

1) Let $\alpha$ be a node on a postorder traversal of $B T(G)$.

2) If $\alpha$ is a leaf or $\alpha$ is a $P^{\prime}$-node then $M \leftarrow \varnothing, U \leftarrow\{\alpha\}$.

3) Else if $\alpha$ is a $N$-node then.

4) If $G[\alpha]=E P_{k}$ or $G[\alpha]=E C_{k}$ then $M \leftarrow$ MAXMATCHEP $P_{k}, E C_{k}, U \leftarrow M$-unsaturated vertices.

5) Else if $G=\overline{E P}_{k}^{\text {bip }}$ then $M \leftarrow$ MAXMATCH $\overline{E P}_{k}^{\text {bip }}, U \leftarrow M$-unsaturated vertices.

6) $\quad$ Else $M \leftarrow$ MAXMATCH$\overline{E C}_{k}^{\text {bip }}, U \leftarrow M$-unsaturated vertices.

7) $\quad$ Replace $\alpha$ by a leaf $\alpha^{\prime}$ together with $M$ and $U$.

8) Else let $\alpha_{1}$ and $\alpha_{2}$ be the two children of $\alpha$ in $B T(G)$.

9) Let $M_{1}$ and $M_{2}$ be respectively the maximum matchings and.

10) $U_{1}$ and $U_{2}$ be respectively the sets of unsaturated vertices of $G\left[\alpha_{1}\right]$ and $G\left[\alpha_{2}\right]$.

11) If $\alpha$ is a $P$-node then $M \leftarrow M_{1} \cup M_{2}, U \leftarrow U_{1} \cup U_{2}$.

12) Else if $\alpha$ is a $K+S$-node then $M \leftarrow M a t c h\left[U_{1}, U_{2}\right], U \leftarrow M$-unsaturated vertices.

13) $\quad$ Else $M \leftarrow \operatorname{Match}\left[U_{1}, U_{2}\right], U \leftarrow M$-unsaturated vertices $M \leftarrow$ Split $\left[U_{1}, U_{2}\right]$, $U \leftarrow M$-unsaturated vertices.

\subsection{Complexity}

We show now that the complexity of our algorithm is $O(n)$.

The total number of Match operations performed by $\operatorname{Match}\left[V_{i}, V_{j}\right]$, is at most $\min \left(\left|V_{i}\right|,\left|V_{j}\right|\right)$. So the run time of step 4 (Procedure MAXMATCH_EP $\_E C_{k}$ ) is $O(n)$.

Consider the steps 5 and 6 which are the Procedures MAXMATCH $\overline{E P}_{k}^{b i p}$ and MAXMATCH $\overline{E C}_{k}^{b i p}$. The variables $l$ and $h$ in Procedure $\operatorname{MATCH}(G)$ assure that the sum of iterations of all while loops in this Procedure is $O\left(n^{\prime}\right)$. Since $n^{\prime}<n$ and the number of Match operation performed by $\operatorname{Match}\left[V_{i}, V_{j}\right]$ is at most $\min \left(\left|V_{i}\right|,\left|V_{j}\right|\right)$ then MATCH $(G)$ runs in $O(n)$ time. The Procedure $M$-unsaturated vertices $(G, M)$ runs in $O(n)$ time since $n^{\prime}<n$.

Since the size of the matching obtained by MATCH $(G)$ is less than or equal to $n / 2$, the construction of the set $M^{\prime}$ and $M^{\prime \prime}$ defined in Procedures Split $\left(M, V_{s}, V_{s+1}\right)$, Split $\left(M, V_{s+1}, V_{s+2}\right)$ and MAXMATCH $\overline{E C}_{k}^{b i p}$, as well as the while loops defined in these Procedures costs $O(n)$ time. So steps 5 and 6 runs in $O(n)$ time.

The total number of Match or Split operations performed in steps 8 to 13 is bounded by the size of maximum matching obtained, which is less or equal to $n / 2$ ([2]), so the run time of steps 8 to 13 is $O(n)$.

Finally, since the number of visited nodes in $B T(G)$ is $O(n)$, this algorithms runs with $O(n)$ time complexity.

\section{Conclusion}

The maximum matching is computed in $O(n)$ time, given a binary canonical decomposition tree of a bipartite $S t a r_{123}$-free graph. The canonical decomposition of a bipartite $\operatorname{Star}_{123}$-free graph can be done in $O(n+m)$ time [14] including the binary canonical decomposition tree construction. Thus, the whole process is in $O(n+m)$ time.

\section{Acknowledgements}

This research is funded by the Deanship of Research and Graduate Studies in Zarqa University/Jordan. The au- 
thor is grateful to anonymous referee's suggestion and improvement of the presentation of this paper.

\section{References}

[1] Lozin, V.V. (2002) Bipartite Graphs without a Skew Star. Discrete Mathematics, 257, 83-100. http://dx.doi.org/10.1016/S0012-365X(01)00471-X

[2] Fouquet, J.L., Giakoumakis, V. and Vanherpe, J.M. (1999) Bipartite Graphs Totally Decomposable by Canonical Decomposition. International Journal of Foundation of Computer Science, 10, 513-533. http://dx.doi.org/10.1142/S0129054199000368

[3] Ben-Dor, A., Karp, R.M., Schwikowski, B. and Shamir, R. (2003) The Restriction Scaf-Fold Problem. Journal of Computational Biology, 10, 385-398. http://dx.doi.org/10.1089/10665270360688084

[4] Buss, S.R. and Yianilos, P.N. (1995) A Bipartite Matching Approach to Approximate String Comparison an Search. Technical Report, NEC Research Institute, Princeton.

[5] Demirci, M.F., Shokoufandeh, A., Keselman, Y., Bretzner, L. and Dickinson, S. (2006) Object Recognition as Manyto-Many Feature Matching. International Journal of Computer Vision, 69, $203-222$. http://dx.doi.org/10.1007/s11263-006-6993-y

[6] Toussaint, G.T. (2004) A Comparison of Rhythmic Similarity Measures. 5th International Conference on Music Information Retrieval, 242-245.

[7] Toussaint, G.T. (2005) The Geometry of Musical Rhythm. Japan Conference on Discrete and Computational Geometry, Berlin-Heidelberg, 198-212. http://dx.doi.org/10.1007/11589440_20

[8] Micali and Vazirani, V.V. (1980) An $O(m \sqrt{n})$ Algorithm for Finding Maximum Matching in General Graphs. FOCS, 17-27.

[9] Moitra and Johnson, R.C. (1989) A Parallel Algorithm for Maximum Matching in Interval Graphs. Proceedings of International Conference on Parallel Processing, 3, 114-120.

[10] Alt, H., Blum, N., Mehlhborn, K. and Paul, M. (1991) Computing a Maximum Cardinality Matching in Bipartite Graphs in Time $O\left(n^{1.5} \sqrt{m \log n}\right)$. Information Processing Letters, 37, 237-240.

http://dx.doi.org/10.1016/0020-0190(91)90195-N

[11] Yu, M.S. and Yang, C.H. (1993) An O(n) Time Algorithm for Maximum Matching on Cographs. Information Processing Letters, 47, 89-93. http://dx.doi.org/10.1016/0020-0190(93)90230-7

[12] Fouquet, J.L., Parfenoff, I. and Thuillier, H. (1997) An O(n) Time Algorithm for Maximum Matching in P4-Tidy Graphs. Information Processing Letters, 62, 281-287. http://dx.doi.org/10.1016/S0020-0190(97)00081-1

[13] Quaddoura, R. (2014) An O(n) Time Algorithm for Maximum Induced Matching In Bipartite Star 123 -Free Graphs. World of Computer Science and Information Technology Journal (WCSIT), 4, 38-41.

[14] Quaddoura, R. (2006) Linear Time Recognition Algorithm of Bipartite Star ${ }_{123}$-Free Graphs. International Arab Journal of Information Technolog, 3, 193-202.

[15] Bondy, J.A. and Murty, U.S.R. (1979) Graph Theory with Applications. North Holland, New York.

[16] Berge, C. (1957) Two Theorems in Graph Theory. Proceedings of the National Academy of Sciences of the United States of America, 43, 842-844. http://dx.doi.org/10.1073/pnas.43.9.842 